\title{
Prealbumin in the diagnosis of bronchopulmonary carcinoid tumours
}

\author{
U R Suresh, S Wilkes, P S Hasleton
}

\begin{abstract}
The reliability of prealbumin as a diagnostic marker was studied in 60 cases of bronchopulmonary carcinoid tumours. There were differences in the incidence of positivity between typical and atypical carcinoids (well differentiated neuroendocrine carcinomas). Seventy five per cent of the carcinoid tumours were positive for prealbumin; (86.7\% typical and $63.3 \%$ atypical carcinoids). In 15 cases, which were Grimelius negative, 10 were prealbumin positive. Only $8.3 \%$ carcinoids were negative with both prealbumin and Grimelius stains. Ten squamous, 10 adeno- and 10 small cell carcinomas showed only occasional scattered prealbumin positive cells.

It is concluded that prealbumin is a useful marker for bronchopulmonary carcinoid tumours. It is cheap, readily available, and should be considered part of routine diagnostic procedures for the diagnosis of carcinoid tumours.
\end{abstract}

Carcinoid tumours produce a variety of proteins including the hormones 5-hydroxytryptamine and neurone specific enolase. Immunohistochemistry can be used to aid diagnosis, but the fact that there are so many different markers indicates there is no single reliable diagnostic indicator for these tumours. With this in mind we investigated the use of prealbumin as a marker for pulmonary carcinoid tumours and other common lung cancers.

Human prealbumin is a serum protein, synthesised predominantly in the liver. Its presence has been previously shown in carcinoid tumours. ${ }^{12}$ Miller et al studied prealbumin staining in 106 carcinoid tumours, including 29 bronchial carcinoids, ${ }^{1}$ and Bussolati et al included four bronchial carcinoids in their study of 37 carcinoids. ${ }^{2}$ Neither of these studies differentiated atypical from typical carcinoids. Our study is one of the largest series of bronchopulmonary carcinoids and shows the prealbumin staining patterns in typical and atypical variants. We also investigated the distribution of prealbumin in other histological types of lung tumours.

\section{Methods}

Formalin fixed, paraffin wax embedded tissues were obtained from fresh lobectomy and pneumonectomy specimens at the Regional
Cardiothoracic Centre, Wythenshawe Hospital, Manchester. A total of 60 cases of bronchopulmonary carcinoid tumours were obtained. All had been diagnosed by haematoxylin and eosin and Grimelius stains. In some, electron microscopy had also been used. Half the cases had been diagnosed as "typical" carcinoids and the rest as "atypical" or "malignant" carcinoids (well differentiated neuroendocrine carcinomas), using previously described criteria. ${ }^{3}$ Cells with a brown staining cytoplasm were counted as positive. We estimated the percentage of positive cells in each case on a $0-30 \%, 31-60 \%$, and $61-100 \%$ scale. We also studied 10 squamous cell, 10 adeno-, and 10 small cell carcinomas. All were obtained at pneumonectomy. The control tissue used was normal endocrine pancreas.

Sections $(5 \mu \mathrm{m})$ were dewaxed, rehydrated, and endogenous peroxidase activity blocked with $0.5 \% \mathrm{H}_{2} \mathrm{O}_{2}$ in methanol. Sections were washed in tap water and then placed in $0.1 \%$ trypsin in calcium chloride $(\mathrm{pH} 7.8)$ at $37^{\circ} \mathrm{C}$ for 15 minutes. The sections were then washed in TRIS buffered saline (TBS) (pH 7.6) and the primary antiserum (Dakopatts rabbit antihuman Prealbumin Code No: A002) applied at a dilution of 1 in 200 and inculated for 45 minutes at room temperature. Subsequent applications of swine antirabbit serum and peroxidase antiperoxidase antiserum were applied at dilutions of 1 in 500 and 1 in 400 , respectively, and incubated for $\mathbf{4 5}$ minutes at room temperature.

Peroxidase activity was shown with diaminobenzidine tetrahydrochloride (Sigma) (10 $\mathrm{mg}$ in $20 \mathrm{ml}$ of diaminobenzidine buffer) and the nuclei counterstained with haematoxylin.

\section{Results}

Seventy five per cent (45 cases) of the 60 carcinoid tumours were positive, either focally or diffusely, for prealbumin. The staining was finely granular and cytoplasmic. There was a small amount of background staining in the stroma in areas of necrosis and haemorrhage, but this did not interfere with interpretation. The remaining $(15 / 60) 25 \%$ of cases were negative.

In the "typical" carcinoids (fig 1), $87 \%$ $(26 / 30)$ were positive with prealbumin and $13 \%(4 / 30)$ were negative. In the "atypical" carcinoids (fig 2) $63 \%$ (19/30) were positive and $37 \%(11 / 30)$ were negative. 


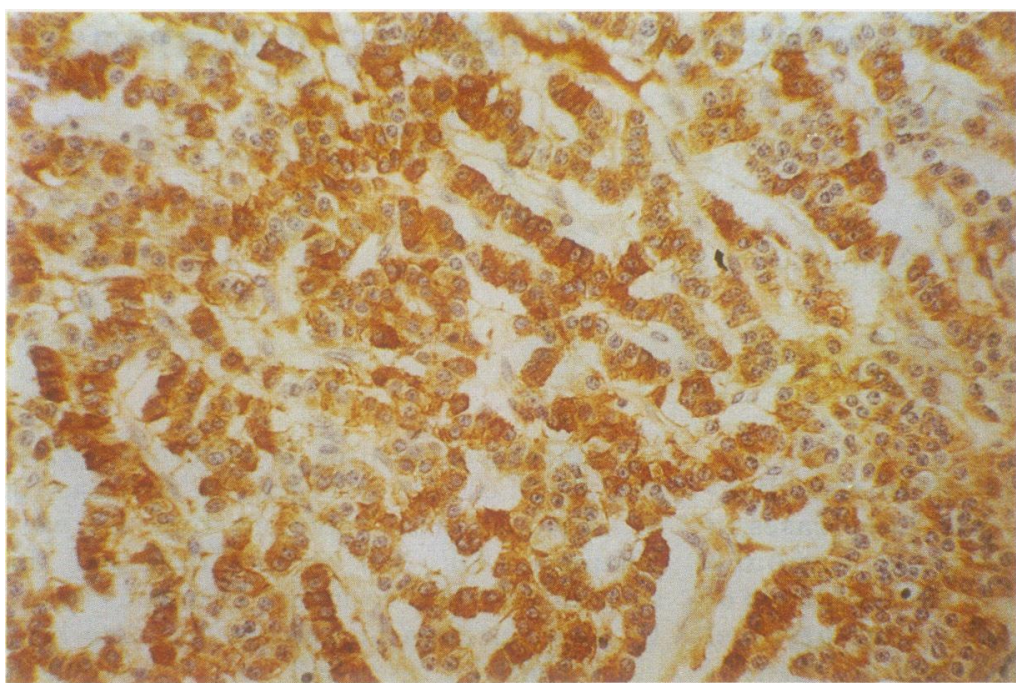

Figure 1 Typical carcinoid with a trabecular pattern showing prominent reactivity with prealbumin (prealbumin).

Of the 26 "typical" carcinoids, 10 cases fell into the $0-30 \%$ group and eight each into the $31-60 \%$ and $61-100 \%$ groups. Of the 19 "atypical" carcinoids, seven were in the $0-30 \%$ group, 10 in the $30-60 \%$ group, and two in the $60-100 \%$ group.

The results of the prealbumin staining were compared with Grimelius staining. Fifteen of the 60 cases were Grimelius negative; in 10 of these prealbumin staining was positive.

All the adenocarcinomas were negative. Half (5/10) squamous carcinomas and two of the 10 small cell carcinomas showed occasional scattered cells staining weakly with prealbumin.

\section{Discussion \\ In routine histopathology the diagnosis of typical carcinoid tumours is usually straight- forward, but in atypical carcinoids the diag- nosis may be more difficult. In many cases the Grimelius stain is of additional value. Blondal et al found all carcinoid tumours were Grimelius positive, ${ }^{4}$ but this is not a universal}

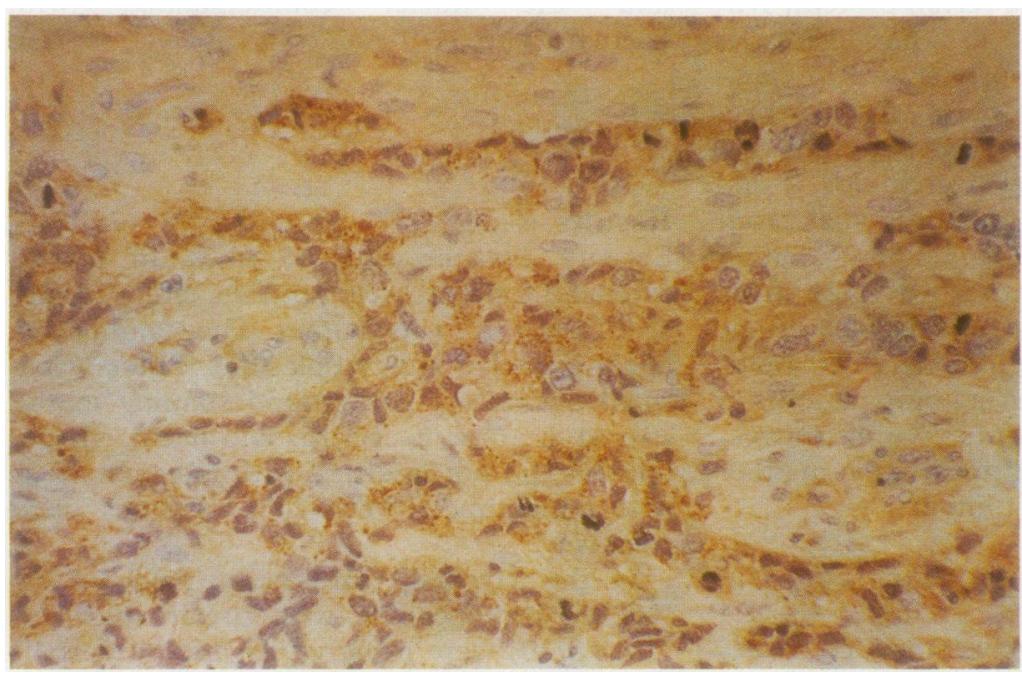

Figure 2 Atypical carcinoid with a disorganised growth pattern (prealbumin). finding. Another stain used for diagnosis of carcinoids is neuron specific enolase (NSE), though this marker has recently been found to be positive in several large cell, squamous, and adenocarcinomas and thus cannot differentiate atypical and typical carcinoids from carcinomas. ${ }^{5}$ Seventy one per cent of small cell carcinomas stained positively with NSE but this figure was less $(35 \%)$ where crush artefact was present. Eighty eight per cent of small cell carcinomas (intermediate cell type) were positive. Seven of $10(70 \%)$ squamous cell carcinomas showed positivity, four of seven $(57 \%)$ adenocarcinomas and three of four $(75 \%)$ large cell carcinomas. ${ }^{5}$ Markers such as chromogranin A and B have also been used to identify neuroendocrine cells. ${ }^{6}$

Several other peptides have been identified in carcinoid tumours. ${ }^{7}$ In a few cases of poorly differentiated carcinoids, however, it can be extremely difficult to identify the neuroendocrine nature of the tumour. Conversely, the diagnosis of "atypical" or "malignant" carcinoids is always difficult. The currently used criteria are cellular pleomorphism, number of mitoses, presence of necrosis, lymphatic or vascular invasion and undifferentiated areas in the presence of a carcinoid growth pattern. ${ }^{8}$ This distinction is important as it relates to prognosis. ${ }^{3}$ Special stains have so far not solved this problem. We therefore tried an immunoperoxidase method using prealbumin in 60 cases of pulmonary carcinoid tumours to determine whether this stain would differentiate the two groups of neuroendocrine tumours.

This seems to be the largest single series of pulmonary carcinoids to be stained with prealbumin. Miller et al, in their study of 106 carcinoid tumours, included 20 bronchial carcinoids and found $79 \%$ of these to be positive for prealbumin. ${ }^{1}$ No attempt was made to differentiate atypical and typical carcinoids. In this study $75 \%(45 / 60)$ of all pulmonary carcinoids were positive for prealbumin. Nineteen of $30(63 \%)$ "atypical" carcinoids were positive but a higher proportion of "typical" carcinoids stained with prealbumin $(87 \%)$. Prealbumin, therefore, does not differentiate typical from atypical carcinoids but is useful as an immunohistochemical marker for the tumour.

Scattered prealbumin positive cells were also found in the three other common pulmonary malignancies except adenocarcinomas indicating the heterogeneity of lung cancer. Our results indicate that prealbumin on its own is not a reliable diagnostic marker for carcinoid tumours, but if used in association with the Grimelius stain it can detect more than $90 \%$ of these tumours. Thus in cases with scattered prealbumin staining it would be necessary to consider the positivity in conjunction with the staining pattern with haematoxylin and eosin and Grimelius and other antisera.

A study by Al-Saffar et al on 57 typical carcinoid tumours of lung showed $66.7 \%$ were bombesin positive, $56.7 \%$ were calcitonin positive, and $50 \%$ were gastrin positive. ${ }^{7}$ Linnoila et al studied four carcinoid tumours of lung and found that $75 \%$ were bombesin positive and $50 \%$ were serotonin positive. 
Bombesin was the most frequently expressed peptide in both studies. In comparison our findings show a significantly higher percentage of positivity $(86.7 \%)$ with prealbumin staining in typical carcinoid tumours. Furthermore, on comparing the cost of these immunohistochemical stains, we found that all of them were at least four to six times more expensive than prealbumin. In conclusion, we believe prealbumin is a cheaper and more reliable initial screening marker for carcinoid tumours.

We thank Mrs K Davis for typing the manuscript.

1 Miller ID, Reid WA, Liddle CN, Horne CHW. Immunolocalisation of prealbumin as a marker for carcinoid localisation of prealbumin as a ma
tumours. J Pathol 1984;143:199-204.

2 Bussolati G, Pappoti M, Sapino A. Binding of antibodies against pre-albumin to intestinal and bronchial carcinoids and to pancreatic endocrine tumours. Virchows Arch । Cell Pathol) 1984;45:15-22.

3 Hasleton PS, Gomm S, Blair V, Thatcher N. Pulmonary carcinoid tumour: a clinicopathological study of 35 cases. BrJ Cancer 1986;54:963-7.

4 Blondal T, Grimelius L, Nou E, Wilander E, Aberg T Argyrophil carcinoid tumours of the lung: incidence, clinical study and follow up of 46 patients. Chest 1980 78:840-4

5 Bergh J, Esscher T, Steinholtz L, Nilsson K, Pahlman S. Immunocytochemical demonstration of neuron-specific enolase (NSE) in human lung cancers. Am J Clin Pathol 1985;84:1-7.

6 Walts AE, Said JW, Shintaku IP, Lloyd RV. Chromogranin as a marker of neuroendocrine cells in cytological material-an immunocytochemical study. $\mathrm{Am} J \mathrm{Clin}$ Pathol 1985;84:273-7.

7 Al-Saffar N, White A, Moore M, Hasleton PS. Immunoreactivity of various peptides in typical and atypical bronchopulmonary carcinoid tumours. $\mathrm{Br} J$ Cancer 1988;58:762-6.

8 Grote TH, Macon WR, Davis B, Greco FA, Johnson DH. Atypical carcinoid of the lung: a distinct clinico-pathoAtypical carcinoid of the lung: a disti
logical entity. Chest 1988;93:370-5.

9 Linnoila RI, Mulshine JL, Steinberg SM, et al. Neuroendocrine differentiation in endocrine and non-endocrine lung carcinomas. Am J Clin Pathol 1988;90:641-52. 